\title{
BMJ Open Using theatre as an arts-based knowledge translation strategy for health-related information: a scoping review protocol
}

\author{
Amanda Hall, ${ }^{1}$ Bradley Furlong, ${ }^{1}$ Andrea Pike, ${ }^{1}$ Gabrielle Logan, ${ }^{1}$ \\ Rebecca Lawrence, ${ }^{1,2}$ Alexandra Ryan, ${ }^{1,3}$ Holly Etchegary, ${ }^{4}$ Todd Hennessey, ${ }^{5}$ \\ Elaine Toomey (iD) ${ }^{6}$
}

To cite: Hall A, Furlong B, Pike $A$, et al. Using theatre as an arts-based knowledge translation strategy for healthrelated information: a scoping review protocol. BMJ Open 2019;9:e032738. doi:10.1136/ bmjopen-2019-032738

- Prepublication history and additional material for this paper are available online. To view these files, please visit the journal online (http://dx.doi org/10.1136/bmjopen-2019032738).

Received 02 July 2019 Revised 11 September 2019 Accepted 19 September 2019

Check for updates

(C) Author(s) (or their employer(s)) 2019. Re-use permitted under CC BY-NC. No commercial re-use. See rights and permissions. Published by BMJ.

For numbered affiliations see end of article.

Correspondence to

Andrea Pike;

andrea.pike@med.mun.ca

\section{ABSTRACT}

Introduction Substantial delays in translating evidence to practice mean that many beneficial and vital advances in medical care are not being used in a timely manner. Traditional knowledge translation (KT) strategies have tended to target academics by disseminating findings in academic journals and at scientific conferences. Alternative strategies, such as theatre-based KT, appear to be effective at targeting broader audiences. The purpose of this scoping review is to collate and understand the current state of science on the use of theatre as a KT strategy. This will allow us to identify gaps in literature, determine the need for a systematic review and develop additional research questions to advance the field.

Methods and analysis This review will follow established scoping review methods outlined by Arksey and 0'Malley in conjunction with enhanced recommendations made by Levac et al. The search strategy, guided by an experienced librarian, will be conducted in PubMed, CINHAL and OVID. Study selection will consist of three stages: (1) initial title and abstract scan by one author to remove irrelevant articles and create a shortlist for double screening, (2) title and abstract scan by two authors, and (3) full-text review by two authors. Included studies will report specifically on the use of theatre as means of KT of health-related information to any target population. Two reviewers will independently extract and chart the data using a standardised data extraction form. Descriptive statistics will be used to produce numerical summaries related to study characteristics, KT strategy characteristics and evaluation characteristics. For those studies that included an evaluation of the theatre production as a KT strategy, we will synthesise the data according to outcome.

Ethics and dissemination Ethical approval was not required for this study. Results will be published in relevant journals, presented at conferences and distributed via social media.

\section{INTRODUCTION}

\section{Evidence practice gap}

Evidence practice gaps, defined as the disparity between research evidence and usual clinical practice, ${ }^{1}$ pose a significant problem for healthcare systems and the quality of
Strengths and limitations of this study

- This protocol will follow the recommended guidance for scoping reviews to ensure accuracy, clarity and reproducibility.

- Our team is a multidisciplinary collaboration between experts in the areas of systematic and scoping reviews and application of knowledge translation strategies and arts-based practices to facilitate high-level discussion between fields to ensure accurate and robust interpretation of the findings.

- The review topic and objectives were codeveloped among relevant knowledge users.

- Due to the broad nature of scoping reviews there will be no quality assessment of included studies.

- Only peer-reviewed literature will be considered in this review.

care they provide to patients. Numerous clinical audits across several areas of healthcare have identified a lack of adherence to clinical practice guidelines, resulting in the underuse of evidence-based interventions or overuse of outdated research. ${ }^{2-6}$ This issue is widespread, and research across multiple countries repeatedly shows that patients are receiving unsuitable and outdated care, which in certain cases can be harmful. ${ }^{7}$ For example, poor staff adherence to evidencebased infection prevention practices and hand hygiene practices in hospitals has been linked with healthcare-associated infections which are associated with patient mortality rates varying from $5 \%$ to $35 \% .^{8-12}$ In a 2011 review, Morris et al identified that 17 years was the most commonly reported average time frame for the translation of health research to practice. $^{13}$ These substantial delays in translating evidence to practice means that many beneficial and vital advances in medical care are not being used in a timely manner. 
This presents a real and direct risk to the quality of care provided to patients and also to overall patient safety. Translating evidence into practice to achieve better patient outcomes is therefore becoming a key priority for many health researchers, heath funders and health systems.

Much of the delay in translation of evidence into practice has been attributed to research waste. ${ }^{2-6}$ In 2009 , it was estimated that about $85 \%$ of research investment was wasted and could have been better spent to ensure the transferability of research to practice. ${ }^{14}{ }^{15}$ In 2016 , a review of what funders, regulators, academic institutions and researchers were doing to address waste found an improvement in the generation of clinically relevant research questions and adherence to standards for research reporting and rigour. ${ }^{16}$ However, less has been done to ensure the translation of research findings to practice. ${ }^{16}$ The authors recommended that researchers should systematically plan to use knowledge translation (KT) strategies to more effectively disseminate their research for knowledge users. ${ }^{16}$

\section{Knowledge translation}

$\mathrm{KT}$ is the use of systematic and iterative processes to translate evidence-based research findings into practice, and has gained traction over the last decade. ${ }^{1718}$ However, the field of KT is still emerging with little consensus on the most effective approaches. This is evidenced by a recent scoping review that identified 592 studies (published in the last 20 years) that used 159 theories, frameworks and models to underpin their KT strategies with very limited information on how these were actually applied. ${ }^{19}$ To provide more clear direction, several KT planning guides have also been developed to help researchers and organisations think about how best to translate research findings for knowledge users. ${ }^{172}$ For example, Ian Graham produced 'Guide to Knowledge Translation Planning at CIHR: Integrated and End-of-Grant Approaches' which helps researchers identify their target knowledge users and their KT goals (eg, to increase awareness, knowledge, or skills or to promote behaviour change) and then consider what KT strategies to use to achieve these goals. ${ }^{17}$

\section{KT strategies}

KT strategies (sometimes referred to as interventions) are overt activities or devices that facilitate or encourage the use of research to achieve clinical practice change. ${ }^{21}$ There are numerous KT strategies to choose from, as evidenced in recent reviews which identified over 30 different strategies within the literature. ${ }^{22-25}$ Traditional KT strategies have tended to focus on publishing research findings in academic journals and presenting results at scientific conferences. This approach is typically used to inform other researchers and academics about the latest advances in health research with the goal of advancing science. However, in recent years, the importance of translating health research across all stakeholder groups has been recognised ${ }^{1726}$ to facilitate better uptake of research into practice and reduction of research waste, and to achieve broader and greater research impact. As such, KT has evolved and broadened in scope to include translation and dissemination of evidence for a wider range of knowledge users (eg, clinicians, policymakers, patients and the public). Since these audiences have different knowledge needs and will use the information for different purposes, alternative KT strategies have been proposed. Alternative KT strategies typically include activities and devices such as plain language summaries, evidence briefs, practice guidelines, educational outreach, mass media, toolkits, opinion leaders or financial incentives. Increasingly, healthcare researchers are also using arts-based KT strategies to translate and disseminate their findings; however, much less is known about how this is used.

\section{Arts-based KT}

Arts-based KT strategies can be broadly grouped into three categories, visual (photographs, drawings), literary (poetry) or performance (eg, theatre, narrative-based arts), and are used to translate key, educative messages to broader audiences. ${ }^{27}$ Arts-based KT is a multidisciplinary approach that brings together professionals with a variety of different expertise. This is a considered a strength that may result in unique and improved ways of disseminating research-based evidence that can appeal to more diverse audiences than traditional scientific presentations and posters. ${ }^{28}$ For example, they are likely to garner more attention, stimulate affective responses and incite discussion and story sharing between those involved. ${ }^{27-29}$ They appear to be especially effective at targeting broader audiences because they are accessible to the general public, can be enjoyed without any particular expertise ${ }^{27}$ and cater to a variety of different learning styles. ${ }^{30}$ For this reason, individuals of different ages, genders, backgrounds and cultures can often relate to these art forms, which may heighten their understanding and acceptance of any message being conveyed. ${ }^{27}$

\section{Theatre-based KT}

Theatre is defined as a presentation or activity that uses drama to engage and entertain an audience. ${ }^{31}$ This medium has been commonly used as an educational tool in other disciplines such as education and sociology for its ability to engage audiences on both affective and cognitive levels, a process that has been found to be central to the success of educational interventions. ${ }^{28}{ }^{32}$ In addition, some theatre productions allow for audience participation which places individuals directly in the context of a specific situation, resulting in stronger emotional responses and attention. ${ }^{33}$ Theatre may be an especially effective KT strategy for public audiences because it is a commonplace and culturally acceptable activity in many countries and communities. ${ }^{34}$ It is also often feasible in low-income areas where other forms of media (eg, television or radio) are inaccessible. For example, Islam $e$ t $a l^{55}$ found that only $0.4 \%$ of people in Bangladesh had access to television, so they used a village theatre production to 
convey information about eclampsia. This study found improved eclampsia knowledge using a pre-post survey. ${ }^{35}$ A previous review of the literature up to 2009 found seven studies that used arts-based methods of drama or theatre for disseminating health research. ${ }^{36}$ The theatre productions were used to impart knowledge about chronic conditions, cancer, HIV, dementia and traumatic brain injury through multisensory mechanisms (ie, by watching, hearing and feeling the message as opposed to solely reading journal publications, pamphlets or conference proceedings). ${ }^{28} 32{ }^{36-38}$ While the review highlighted that theatre was one of the arts-based strategies used in health research it provided only a high-level overview of these studies and thus did not provide sufficient detail about the development of the theatre production, methods of evaluation or the outcome measurement tools used to assess effectiveness. While the interest in KT and using arts-based KT strategies such as theatre continues to grow the details on its intended aim, development, production, implementation and evaluation are still unknown. To date there has not been a review that has focused solely on the arts-based strategy of theatre.

\section{Purpose}

This will be the first review to collate and understand the current state of science on the use of theatre as a KT strategy for dissemination. This review will act as the foundation for a potential new programme of research regarding performance arts-based KT strategies, thus we have chosen to use a scoping review methodology. By doing so, it will allow us to first obtain a broad and general understanding of the use of theatre as a KT strategy for health-related information. We will investigate the types of theatre being used, populations being targeted, messages being conveyed, what outcomes are being assessed and the methods of evaluation. In this way, we can identify current gaps in literature, determine the need for a full systematic review of effectiveness and develop additional research questions and methodologies to advance the field.

\section{METHODS AND ANALYSIS}

To ensure the accuracy and reproducibility of this study, we will follow the six-step scoping review guidance outlined by Arksey and O'Malley ${ }^{39}$ in conjunction with enhanced recommendations to this guidance made by Levac et al. ${ }^{40}$ We will also follow the Preferred Reporting Items for Systematic Review and Meta-Analysis extension for Scoping Reviews checklist to ensure rigour. ${ }^{41}$

\section{Stage 1: developing the research question}

The purpose of this review is to understand the 'state of science' regarding the use of theatre as a KT strategy for health-related information. By this we mean establishing a foundational understanding of how theatre has been used and evaluated as a KT strategy, including target audiences, health topics addressed, types of theatre employed and the research study designs and outcomes assessed. This will enable us to identify the knowledge gaps regarding the use and evaluation methods of theatre as a strategy for KT and provide guidance and suggestions for future research.

To meet our objective of understanding the state of science for the arts-based KT strategy of theatre used in a health research context, we will ask the following questions related to population, concept and context:

\section{Population:}

- What audiences are being targeted? Concept:

- What types of theatre are being used for KT of health information?

- How has the theatre production been developed, produced and implemented?

Context:

- What types of health messages are being conveyed?

- What is the KT aim(s) of theatre (eg, awareness, knowledge, skill development, behaviour change)?

- How has the theatre-based KT strategy been evaluated in terms of outcomes and study design?

\section{Stage 2: identifying relevant studies}

The search strategy for this review was informed by strategies in previous systematic reviews on KT strategies. ${ }^{21} 27$ The search strategy was reviewed and adapted by the research team in collaboration with an experienced librarian to combine the KT string with terms for 'Theatre'. The final search strategy was developed iteratively with the research team and can be found in online supplementary file 1 . The search will be conducted from inception in the following databases: PubMed, CINHAL and OVID. These databases were chosen to capture a comprehensive body of literature from health sciences disciplines. The searches will not be limited by language; for non-English studies a combination of freely available online language translation software programs and consultation with colleagues within our respective institutions will assist with translation to English. Reference lists of key articles will be hand-searched by the review team to capture any papers missed in the electronic searches. The search results will be imported into Covidence review management software ${ }^{42}$ and duplicate citations removed.

\section{Stage 3: study selection}

Study selection will consist of three stages: (1) an initial title and abstract scan by one author to remove irrelevant articles and create a shortlist for double screening, (2) a shortlist of titles/abstracts by two authors, followed by (3) a full-text review by two authors. For duplicate screening, two authors will independently screen each citation and document their results on the review spreadsheet. They will also meet multiple times throughout stage 2 (title/ abstract review) to discuss more complicated criteria as needed. During this process, studies will be coded as 'include', 'exclude' or 'unclear'. Studies marked 'include' or 'unclear' will be retrieved for full-text review 
using Covidence online software. Prior to full-text review, reviewers will meet again to discuss uncertainties for inclusion or exclusion criteria. Studies will be coded in the same way as in title and abstract screening, in preparation for data extraction.

Studies that report specifically on the use of theatre as means of KT of health-related information that is derived from health research sources (published peer-reviewed research or practice guidelines) with any target population (public, patients, workers, care providers) will be included in the review. Theatre productions that are based on information sources not supported by research such as opinion papers or magazine articles in which the supporting research cannot be verified will be excluded. Studies in any language will be included proving an accurate translation can be performed. All study designs will be included ranging from descriptive only studies to evaluation studies (including feasibility, process, effectiveness or cost-related evaluations); only peer-reviewed studies will be included. See online supplementary file 2 for a sample of the inclusion/exclusion form.

\section{Stage 4: charting the data}

A standardised data extraction form will be developed in Excel and pilot tested by the review team to allow reviewers to systematically chart the data. Online supplementary file 3 includes a sample data abstraction chart highlighting the data variables that will be extracted from each of the articles included in the review. These include (1) study characteristics (eg, publication year, country of origin); (2) KT strategy characteristics (the target audience(s), goal(s) and how the theatre productions were developed and implemented); and (3) evaluation characteristics (eg, outcome variables, assessment methods, study designs). Two reviewers will independently extract data on the first $10 \%$ of included studies using the data extraction form. Reviewers will then meet with the lead investigator and discuss any uncertainties encountered during extraction, additional data elements they feel should be included, or any other feedback on the data extraction form to determine if the form needs to be refined. The remaining $90 \%$ of studies will be extracted by one reviewer.

\section{Stage 5: collating, summarising and reporting the results}

We will use descriptive statistics to produce numerical summaries related to study characteristics, KT strategy characteristics and evaluation characteristics. We will provide a more detailed narrative synthesis for the theatre KT strategy, including the development of the theatre script (eg, how the health information was sourced, how key messages were distilled, who was involved in the key message process, how the key messages were integrated into the theatre script, if integrity of the key messages was maintained in the script and details about how long this process took and how much it cost to develop) and production details (eg, how many actors, duration, cost, and so on) as well as enactment details (eg, involvement of consumers or audience). Further, we will use the data extracted to classify the styles of theatre into four artsbased KT categories using the classification schema of arts-based KT strategies as reported by Archibald and colleagues. ${ }^{43}$ This schema aims to provide a description of the arts-based KT strategy based on where it sits along two continuums: passive versus active and ambiguous versus precise. ${ }^{43}$ For those studies that included an evaluation of the theatre production as a KT strategy, we will synthesise the data according to outcome. For example, we will provide a descriptive summary of all studies that evaluated outcomes in two main areas: implementation and effectiveness. Implementation outcomes of KT strategies relate to acceptability, reach, appropriateness, feasibility, fidelity and implementation cost. Effectiveness variables include those relating to KT aims; awareness, knowledge, skills and behaviour change. ${ }^{445}$

This stage of data extraction and summarising will be carried out by two independent reviewers who will compare and consolidate their results through consensus. In cases where there is disagreement regarding data extraction or analysis that cannot be resolved through consensus, a third senior reviewer will help resolve the conflict. The summary of data will highlight the similarities, patterns and differences in the way theatre is being used for the KT of health information as reported in the literature. While details about those studies that evaluated theatre-based KT strategies will be summarised, assessment of quality will not be undertaken as quality assessment is beyond the scope of this review. Considering these results, suggestions for future research evaluating theatre as a KT strategy for disseminating key messages from health research will be discussed.

\section{Stage 6: consultation}

Our team includes representatives from the fields of KT, implementation science, theatre arts, psychology and behaviour change research, clinical trials and health services research. We have codeveloped the topic and research questions for the scoping review with all members of the research team. We will develop a consultation panel including representatives from the Canadian Strategy for Patient-Oriented Research KT National Working Group, KT Canada and the Theatre Arts programmes at Memorial University, the University of Alberta and the University of Toronto. Consultation will pertain to (1) identifying if any important studies were missed in the search strategy, (2) interpreting the findings to ensure validity and that any KT or theatre expert perspectives are represented accurately.

\section{Patient and public involvement}

Members of the public were first involved in this work by way of contributing to a priority setting exercise to select which arts-based KT strategies were important and of interest to the public. Members of the public were consulted to help coproduce the research question by helping to set the eligibility criteria for the population 
and outcome terms of the question, thereby helping to set the scope for the research question. Members of the public will be invited to review a plain language summary, an infographic and short video using Adobe Spark that we will use to present the key findings of the review. These will be disseminated to the public via our social media channels and at local or international public engagement sessions.

\section{ETHICS AND DISSEMINATION}

This will be the first comprehensive review of the use of theatre as a strategy for KT in healthcare settings. It will form the foundation for a future programme of interdisciplinary work between researchers in health services, KT and implementation science, KT change agents, educators in the arts and research-based theatre performers. Ethical approval is not required for this scoping review. The search strategy is planned to be completed by September 2019 and the results by June 2020. We plan to disseminate the results in several ways: publication in relevant journals; presentation at relevant conferences (eg, KT Canada, INVOLVE UK); and via social media using short summaries for non-academic audiences including a plain language summary, an infographic to depict findings and a short video with the research team to explain the state of science on using theatre as a KT strategy.

\section{Author affiliations}

${ }^{1}$ Primary Healthcare Research Unit, Memorial University of Newfoundland, St. John's, Newfoundland and Labrador, Canada

${ }^{2}$ Psychology, Memorial University of Newfoundland, St. John's, Newfoundland and Labrador, Canada

${ }^{3}$ School of Human Kinetics, Memorial University of Newfoundland, St. John's, Newfoundland and Labrador, Canada

${ }^{4}$ Clinical Epidemiology, Memorial University of Newfoundland, St. John's, Newfoundland and Labrador, Canada

${ }^{5}$ School of Fine Arts, Memorial University of Newfoundland-Grenfell Campus, Corner Brook, Newfoundland and Labrador, Canada

${ }^{6}$ Health Behaviour Change Research Group, National University of Ireland Galway, Galway, Ireland

\section{Twitter Elaine Toomey @elainetoomey1}

Acknowledgements We acknowledge Ms Michelle Swab, the librarian at Memorial University, for assisting with adapting the search strategy used in this review. We also acknowledge the Strategy for Patient-Oriented Research (SPOR) Primary Healthcare Research and Integration to Improve Health System Efficiency (PRIIME) initiative for providing research support in the way of four part-time studentships that involve undertaking training to assist with data screening, collection and synthesis on various scoping reviews that will impact better translation of evidence into practice.

Contributors $\mathrm{AH}, \mathrm{ET}$ and $\mathrm{TH}$ conceptualised and designed this scoping review. $A H, E T, B F$ and AP drafted the protocol. AH, GL, RL, AR and BF developed the search strategy and conducted the search. All authors reviewed and provided feedback on the methods and analysis as well as the manuscript. ET, AP and HE reviewed and provided feedback on the manuscript on both content and clarity. All authors provided their approval to publish this manuscript.

Funding The authors have not declared a specific grant for this research from any funding agency in the public, commercial or not-for-profit sectors.

Competing interests None declared.

Patient consent for publication Not required.

Provenance and peer review Not commissioned; externally peer reviewed.
Open access This is an open access article distributed in accordance with the Creative Commons Attribution Non Commercial (CC BY-NC 4.0) license, which permits others to distribute, remix, adapt, build upon this work non-commercially, and license their derivative works on different terms, provided the original work is properly cited, appropriate credit is given, any changes made indicated, and the use is non-commercial. See: http://creativecommons.org/licenses/by-nc/4.0/.

ORCID iD

Elaine Toomey http://orcid.org/0000-0001-5941-0838

\section{REFERENCES}

1 Graham ID, Logan J, Harrison MB, et al. Lost in knowledge translation: time for a MAP? J Contin Educ Health Prof 2006;26:13-24.

2 Chalmers I, Bracken MB, Djulbegovic B, et al. How to increase value and reduce waste when research priorities are set. The Lancet 2014;383:156-65.

3 loannidis JPA, Greenland S, Hlatky MA, et al. Increasing value and reducing waste in research design, conduct, and analysis. The Lancet 2014;383:166-75.

4 Salman RA-S, Beller E, Kagan J, et al. Increasing value and reducing waste in biomedical research regulation and management. The Lancet 2014;383:176-85.

5 Chan A-W, Song F, Vickers A, et al. Increasing value and reducing waste: addressing inaccessible research. The Lancet 2014;383:257-66.

6 Glasziou P, Altman DG, Bossuyt P, et al. Reducing waste from incomplete or unusable reports of biomedical research. The Lancet 2014;383:267-76.

7 Grol R. Successes and failures in the implementation of evidencebased guidelines for clinical practice. Med Care 2001;39:II-46.

8 Flodgren G, Conterno LO, Mayhew A, et al. Interventions to improve professional adherence to guidelines for prevention of device-related infections. Cochrane Database of Systematic Reviews 2013;3.

9 Gammon J, Morgan-Samuel H, Gould D. A review of the evidence for suboptimal compliance of healthcare practitioners to standard/ universal infection control precautions. J Clin Nurs 2008;17:157-67.

10 Pittet D, Donaldson L. Challenging the world: patient safety and health care-associated infection. Int J Qual Health Care 2006;18:4-8.

11 Boyce JM, Pittet D. Guideline for hand hygiene in health-care settings: recommendations of the healthcare infection control practices Advisory Committee and the HICPAC/SHEA/APIC/IDSA hand hygiene Task force. Infect Control Hosp Epidemiol 2002;23(12 Suppl):S3-40.

12 Osborne S. Influences on compliance with standard precautions among operating room nurses. Am J Infect Control 2003;31:415-23.

13 Morris ZS, Wooding S, Grant J. The answer is 17 years, what is the question: understanding time lags in translational research. $J R S O c$ Med 2011;104:510-20.

14 Chen Y-long, Yang K-hu. Avoidable waste in the production and reporting of evidence. The Lancet 2009;374.

15 Chalmers I, Glasziou P. Avoidable waste in the production and reporting of research evidence. The Lancet 2009;374:86-9.

16 Moher D, Glasziou P, Chalmers I, et al. Increasing value and reducing waste in biomedical research: who's listening? The Lancet 2016;387:1573-86.

17 Canadian Institutes of Health Research. Guide to knowledge translation planning at CIHR: integrated and end-of-grant approaches, 2012. Available: http://www.cihr-irsc.gc.ca/e/ documents/kt_Im_ktplan-en.pdf

18 Grimshaw JM, Eccles MP, Lavis JN, et al. Knowledge translation of research findings. Implementation Science 2012;7.

19 Strifler L, Cardoso R, McGowan J, et al. Scoping review identifies significant number of knowledge translation theories, models, and frameworks with limited use. J Clin Epidemiol 2018;100:92-102.

20 Alberta Addiction and Mental Health Research Partnership Program. Knowledge translation evaluation planning guide, 2014. Available: https://www.albertahealthservices.ca/assets/info/res/mhr/if-res-mhrkt-evaluation-guide.pdf

21 Scott SD, Albrecht L, O'Leary K, et al. A protocol for a systematic review of knowledge translation strategies in the allied health professions. Implementation Science 2011;6.

22 Grol R, Grimshaw J. From best evidence to best practice: effective implementation of change in patients' care. The Lancet 2003;362:1225-30.

23 Scott SD, Albrecht L, O'Leary K, et al. Systematic review of knowledge translation strategies in the allied health professions. Implementation Science 2012;7. 
24 Albrecht L, Archibald M, Snelgrove-Clarke E, et al. Systematic review of knowledge translation strategies to promote research uptake in child health settings. J Pediatr Nurs 2016;31:235-54.

25 Effective Practice and Organisation of Care (EPOC). Epoc taxonomy, 2015. Available: https://epoc.cochrane.org/epoc-taxonomy

26 Involve. We believe that people should be at the heart of decisionmaking. Available: http://www.involve.org.uk/

27 Scott SD, Brett-MacLean P, Archibald M, et al. Protocol for a systematic review of the use of narrative storytelling and visual-artsbased approaches as knowledge translation tools in healthcare. Syst Rev 2013;2:19.

28 Parsons JA, Boydell KM. Arts-based research and knowledge translation: some key concerns for health-care professionals. $J$ Interprof Care 2012;26:170-2.

29 Jones K. A biographic researcher in pursuit of an aesthetic: The use of arts-based (re) presentations in "performative" dissemination of life stories. Qualitative Sociology Review 2006;2:66-85.

30 Gardner H. Taking a multiple intelligences (MI) perspective. Behav Brain Sci 2017;40.

31 Theatre. Oxford English dictionary. Available: https://en. oxforddictionaries.com/definition/theatre

32 Colantonio A, Kontos PC, Gilbert JE, et al. After the crash: researchbased Theater for knowledge transfer. J Contin Educ Health Prof 2008;28:180-5.

33 Cheadle A, Cahill C, Schwartz PM, et al. Engaging youth in learning about healthful eating and active living: an evaluation of educational Theater programs. J Nutr Educ Behav 2012;44:160-5.

34 Pelto PJ, Singh R. Community street theatre as a tool for interventions on alcohol use and other behaviors related to HIV risks. AIDS Behav 2010;14:147-57.
35 Islam KS, Sachchu SA, Sandani R, et al. Using village theatre to increase knowledge about eclampsia in Bangladesh. J Obstet Gynaecol Res 2001;27:199-204.

36 Fraser KD, al Sayah F. Arts-based methods in health research: a systematic review of the literature. Arts Health 2011;3:110-45.

37 Gray RE, Sinding C, Fitch MI. Navigating the social context of metastatic breast cancer: reflections on a project linking research to drama. Health 2001;5:233-48.

38 Kontos PC, Naglie G. Expressions of personhood in Alzheimer's: moving from ethnographic text to performing ethnography. Qualitative Research 2006;6:301-17.

39 Arksey H, O'Malley L. Scoping studies: towards a methodological framework. Int J Soc Res Methodol 2005;8:19-32.

40 Levac D, Colquhoun H, O'Brien KK. Scoping studies: advancing the methodology. Implement Sci 2010;5.

41 Tricco AC, Lillie E, Zarin W, et al. PRISMA extension for scoping reviews (PRISMA-ScR): checklist and explanation. Ann Intern Med 2018;169:467-73.

42 Veritas Health Innovation. Covidence systematic review software. Melbourne, Australia. www.covidence.org

43 Archibald MM, Caine V, Scott SD. The development of a classification schema for arts-based approaches to knowledge translation. Worldviews Evid Based Nurs 2014;11:316-24.

44 Peters $\mathrm{DH}$, Adam T, Alonge $\mathrm{O}$, et al. Implementation research: what it is and how to do it. BMJ 2013;347.

45 Glasgow RE, Klesges LM, Dzewaltowski DA, et al. Evaluating the impact of health promotion programs: using the RE-AIM framework to form summary measures for decision making involving complex issues. Health Educ Res 2006;21:688-94. 